\title{
A New Faster Iterative Scheme for Numerical Fixed Points Estimation of Suzuki's Generalized Nonexpansive Mappings
}

\author{
Shanza Hassan, ${ }^{1}$ Manuel De la Sen $\left(\mathbb{D},{ }^{2}\right.$ Praveen Agarwal $\left(\mathbb{D},{ }^{3,4,5}\right.$ Qasim Ali, ${ }^{1}$ \\ and Azhar Hussain $\mathbb{1}^{6,7}$ \\ ${ }^{1}$ Department of Mathematics, University of Sargodha, Sargodha 40100, Pakistan \\ ${ }^{2}$ Institute of Research and Development of Processes IIDP, University of the Basque Country, Campus of Leioa, Leioa, Bizkaia, \\ P.O. Box 48490, Spain \\ ${ }^{3}$ Harish-Chandra Research Institute (HRI), Allahabad, UP, India \\ ${ }^{4}$ Anand International College of Engineering, Jaipur 303012, India \\ ${ }^{5}$ International Center for Basic and Applied Sciences, Jiapur 302029, India \\ ${ }^{6}$ Nonlinear Analysis Research Group, Ton Duc Thang University, Ho Chi Minh City, Vietnam \\ ${ }^{7}$ Faculty of Mathematics and Statistics, Ton Duc Thang University, Ho Chi Minh City, Vietnam
}

Correspondence should be addressed to Azhar Hussain; azharhussain@tdtu.edu.vn

Received 5 January 2020; Accepted 13 April 2020; Published 3 July 2020

Academic Editor: Kishin Sadarangani

Copyright (C) 2020 Shanza Hassan et al. This is an open access article distributed under the Creative Commons Attribution License, which permits unrestricted use, distribution, and reproduction in any medium, provided the original work is properly cited.

The purpose of this paper is to introduce a new four-step iteration scheme for approximation of fixed point of the nonexpansive mappings named as $S^{*}$-iteration scheme which is faster than Picard, Mann, Ishikawa, Noor, Agarwal, Abbas, Thakur, and Ullah iteration schemes. We show the stability of our proposed scheme. We present a numerical example to show that our iteration scheme is faster than the aforementioned schemes. Moreover, we present some weak and strong convergence theorems for Suzuki's generalized nonexpansive mappings in the framework of uniformly convex Banach spaces. Our results extend, improve, and unify many existing results in the literature.

\section{Introduction}

Most of the nonlinear equations can be transformed into a fixed point problem as follows:

$$
\mathscr{F} u=u \text {, }
$$

where $\mathscr{F}$ is a self-map on a certain distance space $\mathscr{X}$ and the solution of the aforementioned equation is considered as a fixed point of the mapping $\mathscr{F}$. Banach [1] proved that if a self-map $\mathscr{F}$ on a complete metric space is such that

$$
d(\mathscr{F} u, \mathscr{F} v) \leq q d(u, v),
$$

for $0 \leq q<1$, then it possesses a unique fixed point $u^{*}$. Moreover, the iterative process

$$
\mathscr{F} u_{n}=u_{n+1} \text {, }
$$

called the Picard iteration process, converges to $u^{*}$. It is worth mentioning that Picard iteration process is useful for the approximation of the fixed point of the contraction mappings but the case when ones dealing with nonexpansive mappings it may fail to converge to the fixed point even if $\mathscr{F}$ has a unique fixed point. Krasnosel'skii [2] showed that Mann [3] iteration process can approximate the fixed points of a nonexpansive mapping. In this iteration scheme, the sequence $\left(u_{n}\right)$ is generated by an arbitrary $u_{0} \in C$ as

$$
u_{n+1}=\left(1-\alpha_{n}\right) u_{n}+\alpha_{n} \mathscr{F} u_{n}, \quad \forall n \geq 0,
$$

where $\left(\alpha_{n}\right)$ is in $(0,1)$.

In 1974, Ishikawa [4] developed an iterative scheme to approximate the fixed point of nonexpansive mappings, where $\left(u_{n}\right)$ is defined iteratively starting from $u_{0} \in C$ by

$$
\left.\begin{array}{l}
u_{n+1}=\left(1-\alpha_{n}\right) u_{n}+\alpha_{n} \mathscr{F} v_{n} \\
v_{n}=\left(1-\beta_{n}\right) u_{n}+\beta_{n} \mathscr{F} u_{n}
\end{array}\right\},
$$

for all $n \geq 0$, where $\left(\alpha_{n}\right)$ and $\left(\beta_{n}\right)$ are in $(0,1)$. 
For the approximation of the fixed point of nonexpansive mappings, Mann and Ishikawa iterative methods have been studied by several authors (see e.g., [5-9]).

Another iteration scheme was proposed by Noor [10] in 2000 , for $u_{0} \in C$, the sequence $\left(u_{n}\right)$ is defined by

$$
\left.\begin{array}{l}
u_{n+1}=\left(1-\alpha_{n}\right) u_{n}+\alpha_{n} \mathscr{F} v_{n} \\
v_{n}=\left(1-\beta_{n}\right) u_{n}+\beta_{n} \mathscr{F} w_{n} \\
w_{n}=\left(1-\gamma_{n}\right) u_{n}+\gamma_{n} \mathscr{F} u_{n}
\end{array}\right\},
$$

for all $n \geq 0$, where $\left(\alpha_{n}\right),\left(\beta_{n}\right)$, and $\left(\gamma_{n}\right)$ are in $(0,1)$.

Agarwal et al. [11], in 2007, proposed the following iterative scheme: for arbitrary $u_{0} \in C$, a sequence $\left\{u_{n}\right\}$ is generated by

$$
\left.\begin{array}{c}
u_{n+1}=\left(1-\alpha_{n}\right) \mathscr{F} u_{n}+\alpha_{n} \mathscr{F} v_{n} \\
v_{n}=\left(1-\beta_{n}\right) u_{n}+\beta_{n} \mathscr{F} u_{n}
\end{array}\right\},
$$

for all $n \geq 0$, where $\left(\alpha_{n}\right)$ and $\left(\beta_{n}\right)$ are in $(0,1)$. They proved that this procedure converges faster than Mann iteration for contraction mappings.

In 2014, Abbas and Nazir [12] developed an iterative scheme which is faster than Agarwal et al.'s [11] scheme, where a sequence $\left(u_{n}\right)$ is formulated from arbitrary $u_{0} \in C$ by

$$
\left.\begin{array}{l}
u_{n+1}=\left(1-\alpha_{n}\right) \mathscr{F} v_{n}+\alpha_{n} \mathscr{F} w_{n} \\
v_{n}=\left(1-\beta_{n}\right) \mathscr{F} u_{n}+\beta_{n} \mathscr{F} w_{n} \\
w_{n}=\left(1-\gamma_{n}\right) u_{n}+\gamma_{n} \mathscr{F} u_{n}
\end{array}\right\},
$$

for all $n \geq 0$, where $\left(\alpha_{n}\right),\left(\beta_{n}\right)$, and $\left(\gamma_{n}\right)$ are in $(0,1)$.

Later in 2016, Thakur et al. [13] developed the following iterative procedure, where a sequence $\left(u_{n}\right)$ is generated iteratively by arbitrary $u_{0} \in C$ and

$$
\left.\begin{array}{l}
u_{n+1}=\left(1-\alpha_{n}\right) \mathscr{F} w_{n}+\alpha_{n} \mathscr{F} v_{n} \\
v_{n}=\left(1-\beta_{n}\right) w_{n}+\beta_{n} \mathscr{F} w_{n} \\
w_{n}=\left(1-\gamma_{n}\right) u_{n}+\gamma_{n} \mathscr{F} u_{n}
\end{array}\right\},
$$

for all $n \geq 0$, where $\left(\alpha_{n}\right),\left(\beta_{n}\right)$, and $\left(\gamma_{n}\right)$ are in $(0,1)$.

Recently, in 2018, Ullah and Arshad developed a new iteration process which converges faster than all the aforementioned process, where the sequence is constructed by taking arbitrary $u_{0} \in C$ and

$$
\left.\begin{array}{l}
u_{n+1}=\mathscr{F} v_{n} \\
v_{n}=\mathscr{F}\left(\left(1-\alpha_{n}\right) w_{n}+\alpha_{n} \mathscr{F} w_{n}\right) \\
w_{n}=\left(1-\beta_{n}\right) u_{n}+\beta_{n} \mathscr{F} u_{n}
\end{array}\right\},
$$

for all $n \geq 0$, where $\left(\alpha_{n}\right),\left(\beta_{n}\right)$, and $\left(\gamma_{n}\right)$ are in $(0,1)$.

Our aim is to introduce a new faster iteration process than those mentioned above and to prove the convergence results for Suzuki's generalized nonexpansive mappings in the context of uniformly convex Banach spaces. We also show that our process is stable analytically. Numerically, we compare the rate of convergence of our iteration process with the existing iteration processes.

\section{Preliminaries}

Throughout this paper, $\mathscr{E}$ is a nonempty closed convex subset of a uniformly convex Banach space $\mathscr{X}, \mathbb{N}$ denotes the set of all positive integers and $F(\mathscr{F})$ denotes the set of all fixed points of $\mathscr{F}$, that is,

$$
F(\mathscr{F}):=\{y: \mathscr{F} y=y\} .
$$

Definition 1 (see [14]). A Banach space $\mathscr{X}$ is said to be uniformly convex if for each $\epsilon \in(0,2]$, there exists a $\delta>0$ such that for all $u, v \in \mathscr{X}$,

$$
\left.\begin{array}{l}
\|u\| \leq 1 \\
\|v\| \leq 1 \\
\|u-v\|>\epsilon
\end{array}\right\} \quad \text { implies }\left\|\frac{u+v}{2}\right\| \leq \delta .
$$

Definition 2 (see [15]). A Banach space $\mathscr{X}$ is said to satisfy Opial property if for each sequence $\left(u_{n}\right)$ in $\mathscr{X}$, converging weakly to $u \in \mathscr{X}$, we have

$$
\limsup _{n \rightarrow \infty}\left\|u_{n}-u\right\|<\limsup _{n \rightarrow \infty}\left\|u_{n}-v\right\|,
$$

for all $v \in \mathscr{X}$ such that $u \neq v$.

Definition 3. A mapping $\mathscr{F}: \mathscr{E} \longrightarrow \mathscr{E}$ is called a contraction if there exists $\alpha \in(0,1)$, such that

$$
\|\mathscr{F} u-\mathscr{F} v\| \leq \alpha\|u-v\|, \quad \text { for all } u, v \in \mathscr{E} .
$$

Definition 4. A mapping $\mathscr{F}: \mathscr{E} \longrightarrow \mathscr{E}$ quasi-nonexpansive if for all $u \in \mathscr{E}$ and $p \in F(\mathscr{F})$ and $\alpha \in(0,1)$, we have

$$
\|\mathscr{F} u-p\| \leq \alpha \mid\|u-p\| \text {. }
$$

Definition 5 (see [16]). A mapping $\mathscr{F}: \mathscr{E} \longrightarrow \mathscr{E}$ is called Suzuki's generalized nonexpansive mapping if for all $u, v \in \mathscr{E}$, we have

$$
\frac{1}{2}\|u-\mathscr{F} u\| \leq \alpha\|u-v\| \quad \text { implies } \quad\|\mathscr{F} u-\mathscr{F} v\| \leq\|u-v\| .
$$

Suzuki [16] proved that the generalized nonexpansive mapping is weaker than nonexpansive mapping and stronger than quasi-nonexpansive mapping and obtained some fixed points and convergence theorems for Suzuki's generalized nonexpansive mappings. Recently, many authors have studied fixed-point theorems for Suzuki's generalized nonexpansive mapping (see, e.g., [17]).

Senter and Dotson [7] introduced a class of mappings satisfying condition $(I)$.

Definition 6. A mapping $\mathscr{F}: \mathscr{E} \longrightarrow \mathscr{E}$ is said to satisfy condition $(I)$, if there exists a nondecreasing function $f:[0, \infty) \longrightarrow[0, \infty)$ with $f(0)=0$ and $f(\delta)>0$ for all 
$\delta>0$ such that $\|u-\mathscr{F} u\| \geq f(d(u, F(\mathscr{F})))$, for all $u \in \mathscr{E}$, where $d(u, F(\mathscr{F}))=\inf _{p \in F(\mathscr{F})}\|u-p\|$.

Proposition 1 (see [16]). Let $\mathscr{F}: \mathscr{E} \longrightarrow \mathscr{E}$ be any mapping. Then,

(i) If $\mathscr{F}$ is nonexpansive, then $\mathscr{F}$ is a Suzuki's generalized nonexpansive mapping.

(ii) If $\mathscr{F}$ is a Suzuki's generalized nonexpansive mapping and has a fixed point, then $\mathscr{F}$ is a quasi-nonexpansive mapping.

(iii) If $\mathscr{F}$ is a Suzuki generalized nonexpansive mapping, then

$$
\|u-\mathscr{F} v\| \leq 3\|\mathscr{F} u-u\|+\|u-v\|, \quad \forall u, v \in \mathscr{E} .
$$

Lemma 1 (see [16]). Suppose $\mathscr{F}: \mathscr{E} \longrightarrow \mathscr{E}$ is Suzuki's generalized nonexpansive mapping satisfying Opial property. If $\left(u_{n}\right)$ converges weakly to $u$ and $\lim _{n \longrightarrow \infty}\left\|\mathscr{F} u_{n}-u_{n}\right\|=0$, then $\mathscr{F} u=u$.

Lemma 2 (see [16]). Let $\mathscr{X}$ be a uniformly convex Banach space and $\mathscr{E}$ be a weakly convex compact subset of $\mathscr{X}$. Assume that $\mathscr{F}: \mathscr{E} \longrightarrow \mathscr{E}$ is Suzuki's generalized nonexpansive mapping. Then, $\mathscr{F}$ has a fixed point.

Lemma 3 (see [18]). Let $\mathscr{X}$ be a uniformly convex Banach space and $\left(t_{n}\right)$ be any real sequence such that $0<p \leq u_{n} \leq q<1$ for all $n \geq 1$. Suppose that $\left(u_{n}\right)$ and $\left(v_{n}\right)$ be any two sequences of $\mathscr{X}$ such that $\limsup _{n \rightarrow \infty}\left\|u_{n}\right\| \leq r$, $\lim \sup _{n \longrightarrow \infty}\left\|v_{n}\right\| \leq r$, and $\lim \sup _{n \longrightarrow \infty}\left\|t_{n} u_{n}+\left(1-t_{n}\right) v_{n}\right\|=$ $r$ hold for some $r \geq 0$. Then, $\lim \sup _{n \rightarrow \infty}\left\|u_{n}-v_{n}\right\|=0$.

Definition 7. (see [19]). Let $\mathscr{X}$ be a Banach space and $\mathscr{E}$ be a nonempty closed convex subset of $\mathscr{X}$. Assume that $\left(u_{n}\right)$ is a bounded sequence in $\mathscr{X}$. For $u \in \mathscr{X}$, we set $r\left(u,\left(u_{n}\right)\right)=\lim \sup _{n \rightarrow \infty}\left\|u_{n}-u\right\|$. The asymptotic radius of $\left(u_{n}\right)$ relative to $\mathscr{E}$ is the set $r\left(\mathscr{E},\left(u_{n}\right)\right)=\inf \left\{r\left(u,\left(u_{n}\right)\right): u \in \mathscr{E}\right\}$ and the asymptotic center of $\left(u_{n}\right)$ relative to $\mathscr{E}$ is given by the following set:

$$
\mathscr{A}\left(\mathscr{E},\left(u_{n}\right)\right)=\left\{u \in \mathscr{E}: r\left(u,\left(u_{n}\right)\right)=r\left(\mathscr{E},\left(u_{n}\right)\right)\right\} .
$$

It is known that, in a uniformly convex Banach space, $\mathscr{A}\left(\mathscr{E},\left(u_{n}\right)\right)$ consists of exactly one point.

Definition 8. (see [20]). Let $\mathscr{X}$ be a Banach space and $\mathscr{F}: \mathscr{X} \longrightarrow \mathscr{X}$. Suppose that $u_{0} \in \mathscr{X}$ and $u_{n+1}=f\left(\mathscr{F}, u_{n}\right)$ define an iteration procedure which gives a sequence of points $\left(u_{n}\right)$ in $\mathscr{X}$. Assume that $\left(x_{n}\right)$ converges to the fixed point $p$. Suppose $\left(v_{n}\right)$ be a sequence in $\mathscr{X}$ and $\left(\epsilon_{n}\right)$ be a sequence in $\mathbb{R}^{+}=[0, \infty)$ given by $\epsilon_{n}=\left\|v_{n+1}-f\left(\mathscr{F}, v_{n}\right)\right\|$. Then, the iteration procedure defined by $u_{n+1}=f\left(\mathscr{F}, u_{n}\right)$ is said to be $\mathscr{F}$-stable or stable with respect to $\mathscr{F}$ if

$$
\lim _{n \longrightarrow \infty} \epsilon_{n}=0 \quad \text { iff } \quad \lim _{n \longrightarrow \infty} v_{n}=p .
$$

Definition 9 (see [21]). Let $\mathscr{X}$ be a Banach space and $\mathscr{F}: \mathscr{X} \longrightarrow \mathscr{X}$. Then, $\mathscr{F}$ is called a contractive mapping on $\mathscr{X}$ if there exist $L \geq 0, a \in[0,1)$ such that for each $u, v \in \mathscr{X}$,

$$
\|\mathscr{F} u-\mathscr{F} v\| \leq L\|u-\mathscr{F} u\|+a\|u-v\| .
$$

By using (7), Osilike [21] established several stability results most of which are generalizations of the results of Rhoades [22] and Harder and Hicks [23].

Definition 10 (see [24]). Let $\mathscr{X}$ be a Banach space and $\mathscr{F}: \mathscr{X} \longrightarrow \mathscr{X}$. Then, $\mathscr{F}$ is called a contractive mapping on $\mathscr{X}$ if there exist $b \in[0,1)$ and a monotone increasing function $\varphi: \mathbb{R}^{+} \longrightarrow \mathbb{R}^{+}$with $\varphi(0)=0$, such that for each $u, v \in \mathscr{X}$,

$$
\|\mathscr{F} u-\mathscr{F} v\| \leq \varphi(\|u-\mathscr{F} u\|)+b\|u-v\| .
$$

Lemma 4 (see [25]). If $\lambda$ is a real number such that $0 \leq \lambda<1$, and $\left(\epsilon_{n}\right)$ is the sequence of positive numbers such that

$$
\lim _{n \longrightarrow \infty} \epsilon_{n}=0
$$

then for any sequence of positive numbers $v_{n}$ satisfying

$$
v_{n+1} \leq \lambda v_{n}+\epsilon_{n}, \quad \text { for } n=1,2, \ldots,
$$

we have

$$
\lim _{n \longrightarrow \infty} v_{n}=0
$$

\section{3. $S^{*}$-Iteration Process}

Throughout this section, $C$ be a nonempty set of a Banach space $\mathscr{X}$, and for all $n \geq 0,\left(\alpha_{n}\right),\left(\beta_{n}\right),\left(\gamma_{n}\right)$ and $\left(\zeta_{n}\right)$ are real sequences in the interval $(0,1)$.

We generate the sequence $\left(u_{n}\right)$ iteratively, taking arbitrary $u_{0} \in C$, by

$$
\left.\begin{array}{l}
u_{n+1}=\mathscr{F}\left(\left(1-\alpha_{n}\right) v_{n}+\alpha_{n} \mathscr{F} v_{n}\right) \\
v_{n}=\mathscr{F}\left(\left(1-\beta_{n}\right) w_{n}+\beta_{n} \mathscr{F} w_{n}\right) \\
w_{n}=\mathscr{F}\left(\left(1-\gamma_{n}\right) x_{n}+\gamma_{n} \mathscr{F} x_{n}\right) \\
x_{n}=\mathscr{F}\left(\left(1-\zeta_{n}\right) u_{n}+\zeta_{n} \mathscr{F} u_{n}\right)
\end{array}\right\} .
$$

First, we show that $S^{*}$-iteration scheme (25) converges faster than all aforementioned iteration schemes for contractive mappings due to Berinde [26] and is stable.

\section{Convergence and Stability Results of $S^{*}$-Iteration Process}

First, we establish convergence results for $S^{*}$-iteration process: 
Theorem 1. Let $\mathscr{X}$ be a Banach space and $\mathscr{E}$ be a nonempty closed convex subset of $\mathscr{X}$. Let $\mathscr{F}$ be a nonexpansive self mapping on $\mathscr{E},\left(u_{n}\right)$ be a sequence defined by (25), and $F(\mathscr{F}) \neq \phi$. Then, $\lim _{n \longrightarrow \infty}\left\|u_{n}-p\right\|$ exists for all $p \in F(\mathscr{F})$.
Proof. Let $p \in F(\mathscr{F})$ for all $n \in \mathbb{N}$. From (16), we have

$$
\begin{aligned}
\left\|x_{n}-p\right\| & =\left\|\mathscr{F}\left(\left(1-\zeta_{n}\right) u_{n}+\zeta_{n} \mathscr{F} u_{n}\right)-p\right\| \\
& \leq\left\|\left(1-\zeta_{n}\right) u_{n}+\zeta_{n} \mathscr{F} u_{n}-p\right\| \\
& \leq\left(1-\zeta_{n}\right)\left\|u_{n}-p\right\|+\zeta_{n}\left\|\mathscr{F} u_{n}-p\right\| \\
& \leq\left(1-\zeta_{n}\right)\left\|u_{n}-p\right\|+\zeta_{n}\left\|u_{n}-p\right\| \\
& =\left\|u_{n}-p\right\|, \\
\left\|w_{n}-p\right\| & =\left\|\mathscr{F}\left(\left(1-\gamma_{n}\right) x_{n}+\gamma_{n} F x_{n}\right)-p\right\| \\
& \leq\left\|\left(1-\gamma_{n}\right) x_{n}+\gamma_{n} \mathscr{F} x_{n}-p\right\| \\
& \leq\left(1-\gamma_{n}\right)\left\|x_{n}-p\right\|+\gamma_{n}\left\|\mathscr{F} x_{n}-p\right\| \\
& \leq\left(1-\gamma_{n}\right)\left\|x_{n}-p\right\|+\gamma_{n}\left\|x_{n}-p\right\| \\
& =\left\|x_{n}-p\right\| \\
& \leq\left\|u_{n}-p\right\|, \\
\left\|v_{n}-p\right\| & =\left\|\mathscr{F}\left(\left(1-\beta_{n}\right) w_{n}+\beta_{n} \mathscr{F} w_{n}\right)-p\right\| \\
& \leq\left\|\left(1-\beta_{n}\right) w_{n}+\beta_{n} \mathscr{F} w_{n}-p\right\| \\
& \leq\left(1-\beta_{n}\right)\left\|w_{n}-p\right\|+\beta_{n}\left\|\mathscr{F} w_{n}-p\right\| \\
& \leq\left(1-\beta_{n}\right)\left\|w_{n}-p\right\|+\beta_{n}\left\|w_{n}-p\right\| \\
& =\left\|w_{n}-p\right\| \\
& \leq\left\|u_{n}-p\right\| .
\end{aligned}
$$

Thus,

$$
\begin{aligned}
\left\|u_{n+1}-p\right\| & =\left\|\mathscr{F}\left(\left(1-\alpha_{n}\right) v_{n}+\alpha_{n} \mathscr{F} v_{n}\right)-p\right\| \\
& \leq\left\|\left(1-\alpha_{n}\right) v_{n}+\alpha_{n} \mathscr{F} v_{n}-p\right\| \\
& \leq\left(1-\alpha_{n}\right)\left\|v_{n}-p\right\|+\alpha_{n}\left\|\mathscr{F} v_{n}-p\right\| \\
& \leq\left(1-\alpha_{n}\right)\left\|u_{n}-p\right\|+\alpha_{n}\left\|v_{n}-p\right\| \\
& \leq\left(1-\alpha_{n}\right)\left\|u_{n}-p\right\|+\alpha_{n}\left\|u_{n}-p\right\| \\
& =\left\|u_{n}-p\right\| .
\end{aligned}
$$

Hence, $\lim _{n \longrightarrow \infty}\left\|u_{n}-p\right\|$ exists for all $p \in F(\mathscr{F})$.

Theorem 2. Let $\mathscr{X}$ a uniformly convex Banach space and $\mathscr{E}$ be a nonempty closed convex subset of $\mathscr{X}$. Let $\mathscr{F}: \mathscr{E} \longrightarrow \mathscr{E}$ be a nonexpansive mapping. Suppose that $\left(u_{n}\right)$ is defined by the iteration process $(25)$ and $F(\mathscr{F}) \neq \phi$. Then, the sequence $\left\{u_{n}\right\}$ converges to a point of $F(\mathscr{F})$ if and only if $\liminf _{n \longrightarrow \infty} d\left(u_{n}, F(\mathscr{F})\right)=0$ $d(u, F(\mathscr{F}))=\inf \{\|u-p\|: p \in F(\mathscr{F})\}$.

Proof. Necessity is obvious. Suppose that $\liminf _{n \longrightarrow \infty} d\left(u_{n}, F(\mathscr{F})\right)=0$. As proved in Theorem 1, $\lim _{n \longrightarrow \infty}\left\|u_{n}-u\right\| \quad$ exists for all $u \in F(\mathscr{F})$, so $\lim _{n \longrightarrow \infty} d\left(u_{n}, F(\mathscr{F})\right)$ exists and $\lim _{\inf _{n \rightarrow \infty}} d\left(u_{n}, F(\mathscr{F})\right)=$ 0 by assumption. Now, we will prove that $\left(u_{n}\right)$ is a Cauchy sequence in $\mathscr{E}$. For given $\epsilon>0$, there exists $N \in \mathbb{N}$ such that for all $n \geq N$,

$$
d\left(u_{n}, F(\mathscr{F})\right)<\frac{\epsilon}{2} .
$$

In particular, $\inf \left\{\left\|u_{N}-p\right\|: p \in F(\mathscr{F})\right\}<(\epsilon / 2)$. Hence, there exists $p^{*} \in F(\mathscr{F})$ such that $\left\|u_{N}-p^{*}\right\|<\epsilon / 2$. Now for all $m, n \geq N$

$$
\left\|u_{m+n}-u_{n}\right\| \leq\left\|u_{m+n}-p^{*}\right\|+\left\|u_{n}-p^{*}\right\| \leq 2\left\|u_{N}-p^{*}\right\|<\epsilon,
$$

which shows that $\left(u_{n}\right)$ is a Cauchy sequence in $\mathscr{E}$. But $\mathscr{E}$ is a closed subset of $\mathscr{X}$, so there exists $p \in \mathscr{E}$ such that $\lim _{n \rightarrow \infty} u_{n}=p$. Now, $\lim _{n \longrightarrow \infty} d\left(u_{n}, F(\mathscr{F})\right)=0$ gives $d(p, F(\mathscr{F}))$ which implies $p \in F(\mathscr{F})$.

Next, we prove that our iteration process is $\mathscr{F}$-stable or stable with respect to $\mathscr{F}$.

Theorem 3. Let $\mathscr{X}$ be a Banach space and $\mathscr{F}: X \longrightarrow X$ be a mapping satisfying (21). Suppose $\mathscr{F}$ has a fixed point p. Let 
$\left(u_{n}\right)$ be a sequence in $\mathscr{X}$ satisfying (9). Then, $S^{*}$-iteration process (9) is $\mathscr{F}$-stable.

Proof. Let $\left(t_{n}\right)$ be an arbitrary sequence in $\mathscr{X}$ and the sequence generated by $(25)$ is $u_{n+1}=f\left(\mathscr{F}, u_{n}\right)$ converging to a unique fixed point $p$ and $\epsilon_{n}=\left\|t_{n+1}-f\left(\mathscr{F}, t_{n}\right)\right\|$. We will prove that $\lim _{n \longrightarrow \infty} \epsilon_{n}=0 \Longleftrightarrow \lim _{n \longrightarrow \infty} t_{n}=p$. Assume that $\lim _{n \rightarrow \infty} \epsilon_{n}=0$ and

$$
\begin{aligned}
\left\|t_{n+1}-p\right\| & =\left\|t_{n+1}-f\left(\mathscr{F}, t_{n}\right)+f\left(\mathscr{F}, t_{n}\right)-p\right\| \\
& \leq\left\|t_{n+1}-f\left(\mathscr{F}, t_{n}\right)\right\|+\left\|f\left(\mathscr{F}, t_{n}\right)-p\right\| \\
& \leq\left\|t_{n+1}-\mathscr{F}\left(\left(1-\alpha_{n}\right) s_{n}+\alpha_{n} \mathscr{F} s_{n}\right)\right\|+\left\|\mathscr{F}\left(\left(1-\alpha_{n}\right) s_{n}+\alpha_{n} \mathscr{F} s_{n}\right)-p\right\| \\
& \leq \epsilon_{n}+b\left[1-\alpha_{n}(1-b)\right]\left\|s_{n}-p\right\| \\
& =\epsilon_{n}+b\left[1-\alpha_{n}(1-b)\right]\left\|\mathscr{F}\left(\left(1-\beta_{n}\right) r_{n}+\beta_{n} \mathscr{F} r_{n}\right)-p\right\| \\
& \leq \epsilon_{n}+b^{2}\left[1-\alpha_{n}(1-b)\right]\left[\left(1-\beta_{n}(1-b)\right]\left\|r_{n}-p\right\|\right. \\
& =\epsilon_{n}+b^{2}\left[1-\alpha_{n}(1-b)\right]\left[\left(1-\beta_{n}(1-b)\right]\left\|\mathscr{F}\left(\left(1-\gamma_{n}\right) v_{n}+\gamma_{n} \mathscr{F} v_{n}\right)-p\right\|\right. \\
& \leq \epsilon_{n}+b^{3}\left[1-\alpha_{n}(1-b)\right]\left[\left(1-\beta_{n}(1-b)\right]\left[1-\gamma_{n}(1-b)\right]\left\|v_{n}-p\right\|\right. \\
& =\epsilon_{n}+b^{3}\left[1-\alpha_{n}(1-b)\right]\left[\left(1-\beta_{n}(1-b)\right]\left[1-\gamma_{n}(1-b)\right]\left\|\mathscr{F}\left(\left(1-\zeta_{n}\right) t_{n}+\zeta_{n} \mathscr{F} t_{n}\right)-p\right\|\right. \\
& \leq \epsilon_{n}+b^{4}\left[1-\alpha_{n}(1-b)\right]\left[( 1 - \beta _ { n } ( 1 - b ) ] [ 1 - \gamma _ { n } ( 1 - b ) ] \left[1-\zeta_{n}(1-b)\left\|t_{n}-p\right\| .\right.\right.
\end{aligned}
$$
$[0,1]$

Since $b \in[0,1)$ and $\left(\alpha_{n}\right),\left(\beta_{n}\right),\left(\gamma_{n}\right)$, and $\left(\zeta_{n}\right)$ are in

$$
\begin{aligned}
& b^{4}\left[1-\alpha_{n}(1-b)\right]\left[\left(1-\beta_{n}(1-b)\right]\left[1-\gamma_{n}(1-b)\right]\right. \\
& \cdot\left[1-\zeta_{n}(1-b)\right] \|<1 .
\end{aligned}
$$

Hence by Lemma 4 , we have $\lim _{n \longrightarrow \infty}\left\|t_{n}-p\right\|=0$, which gives $\lim _{n \longrightarrow \infty} t_{n}=p$. On the other hand, suppose that $\lim _{n \longrightarrow \infty} t_{n}=p$. Then,

$$
\begin{aligned}
\epsilon_{n} & =\left\|t_{n+1}-f\left(\mathscr{F}, t_{n}\right)\right\| \\
& =\left\|t_{n+1}-p+p-f\left(\mathscr{F}, t_{n}\right)\right\| \\
& \leq\left\|t_{n+1}-p\right\|+b\left[\left(1-\alpha_{n}\right)+b \alpha_{n}\right]\left\|s_{n}-p\right\| \\
& =\left\|t_{n+1}-p\right\|+b\left[\left(1-\alpha_{n}\right)+b \alpha_{n}\right]\left\|\mathscr{F}\left(\left(1-\beta_{n}\right) r_{n}+\beta_{n} \mathscr{F} r_{n}\right)-p\right\| \\
& \leq\left\|t_{n+1}-p\right\|+b^{2}\left[\left(1-\alpha_{n}\right)+b \alpha_{n}\right]\left[\left(1-\beta_{n}(1-b)\right]\left\|r_{n}-p\right\|\right. \\
& =\left\|t_{n+1}-p\right\|+b^{2}\left[\left(1-\alpha_{n}\right)+b \alpha_{n}\right]\left(1-\beta_{n}\right)\left\|\mathscr{F}\left(\left(1-\gamma_{n}\right) v_{n}+\gamma_{n} \mathscr{F} v_{n}\right)-p\right\| \\
& \leq\left\|t_{n+1}-p\right\|+b^{3}\left[1-\alpha_{n}(1-b)\right]\left[\left(1-\beta_{n}(1-b)\right]\left[1-\gamma_{n}(1-b)\right]\left\|v_{n}-p\right\|\right. \\
& =\left\|t_{n+1}-p\right\|+b^{3}\left[1-\alpha_{n}(1-b)\right]\left[\left(1-\beta_{n}(1-b)\right]\left[1-\gamma_{n}(1-b)\right]\left\|\mathscr{F}\left(\left(1-\zeta_{n}\right) t_{n}+\zeta_{n} \mathscr{F} t_{n}\right)-p\right\|\right. \\
& \leq\left\|t_{n+1}-p\right\|+b^{4}\left[1-\alpha_{n}(1-b)\right]\left[\left(1-\beta_{n}(1-b)\right]\left[1-\gamma_{n}(1-b)\right]\left[1-\zeta_{n}(1-b)\right]\left\|t_{n}-p\right\| .\right.
\end{aligned}
$$

Taking limit as $n \longrightarrow \infty$ in (34), we get $\lim _{n \longrightarrow \infty} \epsilon_{n}=0$.

Now, we present an example to compare the rate of convergence of our iteration scheme with others.

Example 1. Let $\mathscr{X}=\mathbb{R}$ and $C=[1,50]$. Let $\mathscr{F}: C \longrightarrow C$ be a mapping defined by $\mathscr{F} u=\sqrt{u^{2}-9 u+54}$ for all $u \in C$. For $u_{1}=30$ and $\alpha_{n}=\beta_{n}=\gamma_{n}=3 / 4, n=1,2,3, \ldots$..From Table 1 , we can see that all the iteration procedures are converging to $p^{*}=6$. Clearly, our iteration process requires the least number of iteration as compared to other iteration schemes.

In Figure 1, black curve represents our iteration process. The graphical view shows that our iteration process requires less number of iterations as compared to the other iteration processes. The number of iterations in which these processes attain the fixed point is given in Table 2:

\section{Some Convergence Results for Suzuki's Generalized Nonexpansive Mappings}

This section contains some weak and strong convergence results for a sequence generated by $S^{*}$-iteration process for Suzuki generalized nonexpansive mappings in the setting of uniformly convex Banach spaces. 
TABLE 1: Comparison of the rate of convergence with various iteration schemes.

\begin{tabular}{|c|c|c|c|c|c|c|c|c|}
\hline Step & Picard & Ishikawa & Noor & Agarwal & Abbas & Thakur & K. Ullah & $\mathrm{S}^{*}$-iter. \\
\hline 1 & 30.00000000 & 30.00000000 & 30.00000000 & 30.00000000 & 30.00000000 & 30.00000000 & 30.00000000 & 30.00000000 \\
\hline 2 & 26.15339366 & 25.01198240 & 23.48910332 & 24.05033082 & 22.61079008 & 21.30667585 & 17.14034293 & 7.939900241 \\
\hline 3 & 22.41917610 & 20.25475590 & 17.46681907 & 18.43727194 & 15.82815627 & 13.58899597 & 7.920241534 & 6.000499545 \\
\hline 4 & 18.83737965 & 15.85090878 & 12.32658573 & 13.39382036 & 10.25820641 & 8.112973955 & 6.038818684 & 6.000000071 \\
\hline 5 & 15.46966242 & 12.01330515 & 8.727576617 & 9.372555587 & 7.001837925 & 6.225674626 & 6.000469229 & 6.000000000 \\
\hline 6 & 12.41303724 & 9.068862033 & 6.958571160 & 6.993935718 & 6.119154210 & 6.015130221 & 6.000005614 & 6.000000000 \\
\hline 7 & 9.816626625 & 7.282040026 & 6.310214626 & 6.186206786 & 6.011213258 & 6.000960494 & 6.000000067 & 6.000000000 \\
\hline 8 & 7.875056741 & 6.466803146 & 6.097925567 & 6.028369366 & 6.001024303 & 6.000060749 & 6.000000001 & 6.000000000 \\
\hline 9 & 6.718705828 & 6.160065238 & 6.030680 & 6.0041338 & 6.000093304 & 6.000003 & 6.000000000 & 6.000000000 \\
\hline 10 & 6.218734240 & & & & & & & 6.000000000 \\
\hline 11 & 6.058386534 & 6.017902837 & 6.002995608 & 6.000086472 & 6.000000774 & 6.000000016 & 6.000000000 & 6.000000000 \\
\hline 12 & 6.014862308 & 6.005951431 & 6.000935492 & 6.000012498 & 6.000000071 & 6.000000 & 6.000000 & 6.000000000 \\
\hline 13 & 6.003 & 6.00 & & 6.00 & 6.000 & 6.000 & & 0000 \\
\hline 14 & & & & & & & & 000000 \\
\hline 15 & 6.000233641 & 6.000 & 6.0000 & & & & & 6.000000000 \\
\hline 16 & 000058415 & 6.000072376 & 6.0000088 & 6.00 & 6.0000000 & 6.000 & 6.000 & 0000 \\
\hline 17 & 6.0000 & 6.00 & 6.00 & 6.00 & 6.00 & & & 0000 \\
\hline 18 & 6.000003651 & 6.000007979 & 6.000000866 & 6.000 & 6.000000000 & & & 6.000000000 \\
\hline 19 & 6.000000912 & 6.000002649 & 6.000000270 & 6.000 & 6.000000000 & 6.000000000 & 6.000000000 & 6.000000000 \\
\hline 20 & 6.000000227 & 6.000000880 & 6.000000084 & 6.000000000 & 6.000000000 & 6.000000000 & 6.000000000 & 6.000000000 \\
\hline 21 & 6.000000057 & 6.000000293 & 6.000000026 & 6.000000000 & 6.000000000 & 6.000000000 & 6.000000000 & 6.000000000 \\
\hline 22 & 6.000000014 & 6.000000097 & 6.000000008 & 6.000000000 & 6.000000000 & 6.000000000 & 6.000000000 & 6.000000000 \\
\hline 23 & 6.000000003 & 6.000000032 & 6.000000003 & 6.000000000 & 6.000000000 & 6.000000000 & 6.000000000 & 6.000000000 \\
\hline 24 & 6.000000001 & 6.000000010 & 6.000000001 & 6.000000000 & 6.000000000 & 6.000000000 & 6.000000000 & 6.000000000 \\
\hline 25 & 6.000000000 & & & & & & & 6.000000000 \\
\hline 26 & 6.000000000 & 6.000000001 & 6.000000000 & 6.000000000 & 6.000000000 & 6.000000000 & 6.000000000 & 6.000000000 \\
\hline 27 & 6.000000000 & 6.000000000 & 6.000000000 & 6.000000000 & 6.000000000 & 6.000000000 & 6.000000000 & 6.000000000 \\
\hline 28 & 6.000000000 & 6.000000000 & 6.000000000 & 6.000000000 & 6.000000000 & 6.000000000 & 6.000000000 & 6.000000000 \\
\hline 29 & 6.000000000 & & & & & & & 6.000000000 \\
\hline 30 & 6.000000000 & 6.000000000 & 6.000000000 & 6.000000000 & 6.000000000 & 6.000000000 & 6.000000000 & 6.000000000 \\
\hline
\end{tabular}

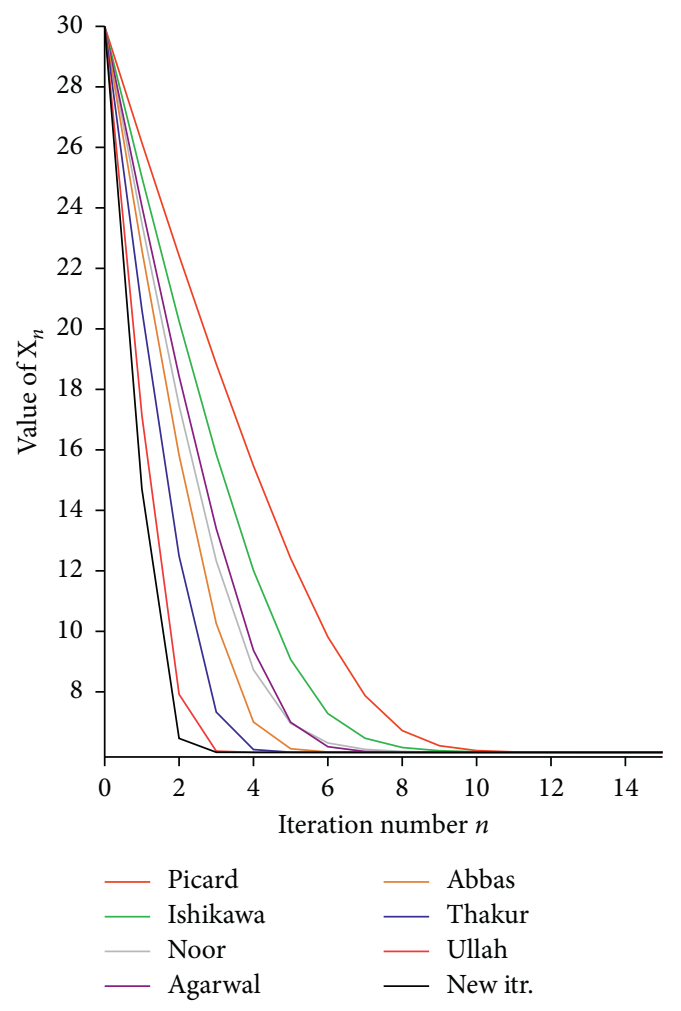

FIgURE 1: Graphical representation of convergence of iterative schemes.
TABLE 2: Number of iterations in which fixed point attains.

\begin{tabular}{lc}
\hline Iterative method & Number of iterations \\
\hline Picard & 25 \\
Ishikawa & 27 \\
Noor & 25 \\
Agarwal & 18 \\
Abbas & 15 \\
Thakur & 13 \\
K. Ullah & 9 \\
S*-iter. $^{*}$ & 5 \\
\hline
\end{tabular}

Lemma 5. Suppose that $\mathscr{E}$ be a nonempty closed convex subset of a Banach space $\mathscr{X}$. Let $\mathscr{F}: \mathscr{E} \longrightarrow \mathscr{E}$ be a Suzuki generalized nonexpansive mapping with $F(\mathscr{F}) \neq \phi$. For $u_{0} \in \mathscr{E}$, the sequence $\left(u_{n}\right)$ generated by $S^{*}$-iteration process, $\lim _{n \longrightarrow \infty}\left\|u_{n}-p\right\|$ exists for all $p \in F(\mathscr{F})$.

Proof. Result follows from Proposition 1(i) and Theorem 1.

Lemma 6. Suppose that $\mathscr{E}$ be a nonempty closed convex subset of a uniformly Banach space $\mathscr{X}$. Let $\mathscr{F}: \mathscr{E} \longrightarrow \mathscr{E}$ be a Suzuki's generalized nonexpansive mapping with $F(\mathscr{F}) \neq \phi$. For arbitrarily chosen $u_{0} \in \mathscr{E}$, the sequence $\left(u_{n}\right)$ is generated by $S^{*}$-iteration process. Then, $F(\mathscr{F}) \neq \phi$ if and only if $\left(u_{n}\right)$ is bounded and $\lim _{n \longrightarrow \infty}\left\|\mathscr{F} u_{n}-u_{n}\right\|=0$. 
Proof. Suppose that $F(\mathscr{F}) \neq \phi$ and let $p \in \mathscr{E}$. Then, by Lemma $5, \lim _{n \longrightarrow \infty}\left\|u_{n}-p\right\|$ exists and $\left(u_{n}\right)$ is bounded. Let

$$
\lim _{n \rightarrow \infty}\left\|u_{n}-p\right\|=r
$$

From (26) and (37), we have

$$
\underset{n \longrightarrow \infty}{\limsup }\left\|u_{n}-p\right\| \leq \underset{n \longrightarrow \infty}{\limsup }\left\|u_{n}-p\right\|=r .
$$

By the Proposition 1 (iii), we have

$$
\begin{aligned}
\underset{n \longrightarrow \infty}{\limsup } \| & \mathscr{F} x_{n}-p\left\|\leq \limsup _{n \longrightarrow \infty}\right\| u_{n}-p \|=r, \\
\left\|u_{n+1}-p\right\| & =\left\|\mathscr{F}\left(\left(1-\alpha_{n}\right) v_{n}+\alpha_{n} \mathscr{F} v_{n}\right)-p\right\| \\
& \leq\left\|\left(1-\alpha_{n}\right) v_{n}+\alpha_{n} \mathscr{F} v_{n}-p\right\| \\
& \leq\left\|v_{n}-p\right\| \\
& =\left\|\mathscr{F}\left(\left(1-\beta_{n}\right) w_{n}+\beta_{n} \mathscr{F} w_{n}\right)-p\right\| \\
& \leq\left\|w_{n}-p\right\| \\
& =\left\|\mathscr{F}\left(\left(1-\gamma_{n}\right) x_{n}+\gamma_{n} \mathscr{F} x_{n}\right)-p\right\| \\
& \leq\left(1-\gamma_{n}\right)\left\|u_{n}-p\right\|+\gamma_{n}\left\|x_{n}-p\right\| \\
& =\left\|u_{n}-p\right\|-\gamma_{n}\left\|u_{n}-p\right\|+\gamma_{n}\left\|x_{n}-p\right\| .
\end{aligned}
$$

This implies that

$$
\begin{aligned}
& \frac{\left\|u_{n+1}-p\right\|-\left\|u_{n}-p\right\|}{\gamma_{n}} \leq\left[\left\|x_{n}-p\right\|-\left\|u_{n}-p\right\|\right], \\
& \left\|u_{n+1}-p\right\|-\left\|u_{n}-p\right\| \leq \frac{\left\|u_{n+1}-p\right\|-\left\|u_{n}-p\right\|}{\gamma_{n}} \\
& \leq\left[\left\|x_{n}-p\right\|-\left\|u_{n}-p\right\|\right], \\
& \left\|u_{n+1}-p\right\| \leq\left\|x_{n}-p\right\|, \\
& r \leq \liminf _{n \rightarrow \infty}\left\|x_{n}-p\right\|, \\
& \lim _{n \longrightarrow \infty}\left\|x_{n}-p\right\|=r, \\
& \lim _{n \longrightarrow \infty}\left\|\left(1-\zeta_{n}\right) u_{n}+\zeta_{n} \mathscr{F}\left(u_{n}\right)-p\right\|=r, \\
& \lim _{n \rightarrow \infty}\left\|\left(1-\zeta_{n}\right)\left(u_{n}-p\right)+\zeta_{n}\left(\mathscr{F}\left(u_{n}\right)-p\right)\right\|=r \text {. }
\end{aligned}
$$

From equations (26) and (37) and Lemma 3, we have

$$
\lim _{n \longrightarrow \infty}\left\|\mathscr{F} u_{n}-u_{n}\right\|=0 \text {. }
$$

Conversely, assume that $\left(u_{n}\right)$ is bounded and $\lim _{n \longrightarrow \infty}\left\|\mathscr{F} u_{n}-u_{n}\right\|=0$. Suppose that $p \in \mathscr{A}\left(\mathscr{E},\left(u_{n}\right)\right)$. Using Proposition 1 (iii), we get

$$
\begin{aligned}
r\left(\mathscr{F} p,\left(u_{n}\right)\right) & =\underset{n \longrightarrow \infty}{\limsup }\left\|u_{n}-\mathscr{F} p\right\| \\
& \leq \limsup _{n \longrightarrow \infty}\left[3\left\|\mathscr{F} u_{n}-u_{n}\right\|+\left\|u_{n}-p\right\|\right] \\
& \leq \underset{n \longrightarrow \infty}{\limsup }\left\|u_{n}-p\right\| \\
& =r\left(p,\left(u_{n}\right)\right) .
\end{aligned}
$$

This shows that $\mathscr{F} p \in \mathscr{A}\left(\mathscr{E},\left(u_{n}\right)\right.$. Since $\mathscr{X}$ is uniformly convex, $\mathscr{A}\left(\mathscr{E},\left(u_{n}\right)\right.$ is singleton. Thus, we have $\mathscr{F} p=p$, that is, $F(\mathscr{F}) \neq \phi$.

Theorem 4 (weak convergence theorem). Suppose that $\mathscr{E}$ be a nonempty closed convex subset of a uniformly Banach space $\mathscr{X}$ with the Opial property. Let $\mathscr{F}: \mathscr{E} \longrightarrow \mathscr{E}$ be Suzuki's generalized nonexpansive mapping. For arbitrarily chosen $u_{0} \in \mathscr{E}$, let the sequence $\left(u_{n}\right)$ be generated by $S^{*}$-iteration process with $F(\mathscr{F}) \neq \phi$. Then, $\left(u_{n}\right)$ converges weakly to a fixed point of $\mathscr{F}$.

Proof. Since $F(\mathscr{F}) \neq \phi$, by Lemma 6 , the sequence $\left(u_{n}\right)$ is bounded and $\lim _{n \longrightarrow \infty}\left\|\mathscr{F} u_{n}-u_{n}\right\|=0$. Also, as $\mathscr{X}$ is uniformly convex so $\mathscr{X}$ is reflexive, thus by Eberlin's theorem, there exists a subsequence of $\left(u_{n}\right)$ say $\left(u_{n_{i}}\right)$ which converges weakly to some $q_{1} \in \mathscr{X}$. Now, since $\mathscr{E}$ is closed and convex so by Mazur's theorem $q_{1} \in \mathscr{E}$. Hence, by Lemma 1 , $q_{1} \in F(\mathscr{F})$. We show that $\left(u_{n}\right)$ converges weakly to $q_{1}$. On contrary, suppose that it is not true. Then, there must exist a subsequence of $\left(u_{n}\right)$, say $\left(u_{n_{j}}\right)$, such that $\left(u_{n_{j}}\right)$ converges weakly to $q_{2} \in \mathscr{E}$ with $q_{1} \neq q_{2}$. Using Lemma 1 , we have $q_{2} \in F(\mathscr{F})$. Now, since $\lim _{n \rightarrow \infty}\left\|u_{n}-p\right\|$ exists for all $p \in F(\mathscr{F})$. Using Lemma 6 and Opial property, we have

$$
\begin{aligned}
\lim _{n \longrightarrow \infty}\left\|u_{n}-q_{1}\right\| & =\lim _{i \rightarrow \infty}\left\|u_{n_{i}}-q_{1}\right\| \\
& <\lim _{i \longrightarrow \infty}\left\|u_{n_{i}}-q_{2}\right\| \\
& =\lim _{n \longrightarrow \infty}\left\|u_{n}-q_{2}\right\| \\
& =\lim _{j \longrightarrow \infty}\left\|u_{n_{j}}-q_{2}\right\| \\
& <\lim _{j \longrightarrow \infty}\left\|u_{n_{j}}-q_{1}\right\| \\
& =\lim _{n \longrightarrow \infty}\left\|u_{n}-q_{1}\right\|,
\end{aligned}
$$

which is a contradiction; hence, $q_{1}=q_{2}$. This shows that $\left(u_{n}\right)$ converges weakly to a fixed point of $\mathscr{F}$.

Theorem 5 (strong convergence theorem). Suppose that $\mathscr{E}$ be a nonempty closed convex subset of a uniformly Banach space $\mathscr{X}$. Let $\mathscr{F}: \mathscr{E} \longrightarrow \mathscr{E}$ be a Suzuki's generalized nonexpansive mapping. For arbitrarily chosen $u_{0} \in \mathscr{E}$, let the sequence $\left(u_{n}\right)$ be generated by $S^{*}$-iteration process with $F(\mathscr{F}) \neq \phi$. Then, $\left(u_{n}\right)$ converges strongly to a fixed point of $\mathscr{F}$.

Proof. Using Lemma 2, we get $F(\mathscr{F}) \neq \phi$ and hence by Lemma 6, we have $\lim _{n \longrightarrow \infty}\left\|\mathscr{F} u_{n}-u_{n}\right\|=0$. By the compactness of $\mathscr{E}$, there exists a subsequence of $\left(u_{n}\right)$, say $\left(u_{n_{i}}\right)$, converging strongly to $p$ for some $p \in \mathscr{E}$. Now by using Proposition 1 (iii), we get

$$
\left\|u_{n_{i}}-\mathscr{F} p\right\| \leq 3\left\|\mathscr{F} u_{n_{i}}-u_{n_{i}}\right\|+\left\|u_{n_{i}}-p\right\| .
$$

Taking limit $i \longrightarrow \infty$, we get $\mathscr{F} p=p$, that is, $p \in F(\mathscr{F})$. By using Lemma $5, \lim n \longrightarrow \infty\left\|u_{n}-p\right\|$ exists for all $p \in F(\mathscr{T})$; hence, $\left\{u_{n}\right\}$ converges strongly to $p$. 
Theorem 6. Suppose that $\mathscr{E}$ be a nonempty closed convex subset of a uniformly Banach space $\mathscr{X}$. Let $\mathscr{F}: \mathscr{E} \longrightarrow \mathscr{E}$ be a Suzuki's generalized nonexpansive mapping. For arbitrarily chosen $u_{0} \in \mathscr{E}$, the sequence $\left(u_{n}\right)$ be generated by $S^{*}$-iteration process with $F(\mathscr{F}) \neq \phi$. If $\mathscr{F}$ satisfies condition $(I)$, then $\left(u_{n}\right)$ converges strongly to a fixed point of $\mathscr{F}$.

Proof. By Lemma 5, $\lim n \longrightarrow \infty\left\|u_{n}-p\right\|$ exists for all $p \in F(\mathscr{F})$; hence, $\quad \lim _{n \longrightarrow \infty} d\left(u_{n}, F(\mathscr{F})\right)$ exists. Let $\lim _{n \longrightarrow \infty}\left\|u_{n}-p\right\|=\delta$ for some $\delta \geq 0$. Now if $\delta=0$, then there is nothing to prove. Suppose $\delta>0$; from condition (I) and the hypothesis, we have

$$
f\left(d\left(u_{n}, F(\mathscr{F})\right)\right) \leq\left\|\mathscr{F} u_{n}-u_{n}\right\| .
$$

As $\quad F(\mathscr{F}) \neq \phi$, by Lemma 5 , we have $\lim _{n \longrightarrow \infty}\left\|\mathscr{F} u_{n}-u_{n}\right\|=0$. Hence, (46) implies that

$$
\lim _{n \longrightarrow \infty} f\left(d\left(u_{n}, F(\mathscr{F})\right)\right)=0 .
$$

Since $f$ is a nondecreasing function, by equation (47), we get $\lim _{n \longrightarrow \infty}\left(d\left(u_{n}, F(\mathscr{F})\right)=0\right.$. Thus, we have a subsequence $\left(u_{n_{i}}\right)$ of $\left(u_{n}\right)$ and a sequence $\left(y_{i}\right)$ in $F(\mathscr{F})$ such that

$$
\left\|u_{n_{i}}-y_{i}\right\|<\frac{1}{2^{i}}, \quad \text { for all } i \in \mathbb{N} \text {. }
$$

From equation (48),

$$
\begin{aligned}
\left\|u_{n_{i+1}}-y_{i}\right\| & \leq\left\|u_{n_{i}}-y_{i}\right\|<\frac{1}{2^{i}}, \\
\left\|y_{i+1}-y_{i}\right\| & \leq\left\|y_{i+1}-u_{i+1}\right\|+\left\|u_{i+1}-y_{i}\right\| \\
& \leq \frac{1}{2^{i+1}}+\frac{1}{2^{i}} \\
& <\frac{1}{2^{i-1}} .
\end{aligned}
$$

Letting $i \longrightarrow \infty$, we get $1 / 2^{i-1} \longrightarrow 0$. Hence, $\left\{y_{i}\right\}$ is a Cauchy sequence in $F(\mathscr{F})$, so it converges to $p$. As $F(\mathscr{F})$ is closed, $p \in F(\mathscr{F})$ and then $\left(u_{n_{i}}\right)$ converges strongly to $p$. Since $\lim _{n \longrightarrow \infty}\left\|u_{n}-p\right\|$ exists, we have $u_{n} \longrightarrow p \in F(\mathscr{F})$. This completes the proof.

\section{Data Availability}

No data were used to support this study.

\section{Conflicts of Interest}

The authors declare that they have no conflicts of interest.

\section{Authors' Contributions}

All authors have contributed equally.

\section{Acknowledgments}

The authors are very grateful to the Basque Government for its support through Grant IT1207-19.

\section{References}

[1] S. Banach, "Sur les opérations dans les ensembles abstraits et leur application aux équations intégrales," Fundamenta Mathematicae, vol. 3, pp. 133-181, 1922.

[2] M. A. Krasnosel'skii, "Two remarks on the method of successive approximations," Uspekhi Matematicheskikh Nauk, vol. 10, pp. 123-127, 1995.

[3] W. R. Mann, "Mean value methods in iteration," Proceedings of the American Mathematical Society, vol. 4, no. 3, p. 506, 1953.

[4] S. Ishikawa, "Fixed points by a new iteration method," Proceedings of the American Mathematical Society, vol. 44, no. 1, p. $147,1974$.

[5] S. Ishikawa, "Fixed points and iteration of a nonexpansive mapping in a Banach space," Proceedings of the American Mathematical Society, vol. 59, no. 1, p. 65, 1976.

[6] S. Reich, "Weak convergence theorems for nonexpansive mappings in Banach spaces," Journal of Mathematical Analysis and Applications, vol. 67, no. 2, pp. 274-276, 1979.

[7] H. F. Senter and W. G. Dotson, "Approximating fixed points of nonexpansive mappings," Proceedings of the American Mathematical Society, vol. 44, no. 2, p. 375, 1974.

[8] K. K. Tan and H. K. Xu, "Approximating fixed points of nonexpansive mappings by the Ishikawa iteration process," Journal of Mathematical Analysis and Applications, vol. 178, no. 2, pp. 301-308, 1993.

[9] L.-C. Zeng, "A note on approximating fixed points of nonexpansive mappings by the Ishikawa iteration process," Journal of Mathematical Analysis and Applications, vol. 226, no. 1, pp. 245-250, 1998.

[10] M. A. Noor, "New approximation schemes for general variational inequalities," Journal of Mathematical Analysis and Applications, vol. 251, no. 1, pp. 217-229, 2000.

[11] R. P. Agarwal, D. O' Regan, and D. R. Sahu, "Lterative construction of fixed points of nearly asymptotically nonexpansive mappings," Journal of Nonlinear and Convex Analysis, vol. 8, no. 1, pp. 61-79, 2007.

[12] M. Abbas and T. Nazir, "A new faster iteration process applied to constrained minimation and feasibility problems," Matematicki Vesnik, vol. 66, no. 2, pp. 223-234, 2014.

[13] B. Thakur, D. Thakur, and M. Postolache, "A new iteration scheme for approximating fixed points of nonexpansive mappings," Filomat, vol. 30, no. 10, pp. 2711-2720, 2016.

[14] K. Goebel and W. A. Kirk, Topic in Metric Fixed Point Theory, Cambridge University Press, Cambridge, UK, 1990.

[15] Z. Opial, "Weak convergence of the sequence of successive approximations for nonexpansive mappings," Bulletin of the American Mathematical Society, vol. 73, pp. 595-597, 1967.

[16] T. Suzuki, "Fixed point theorems and convergence theorems for some generalized nonexpansive mappings," Journal of Mathematical Analysis and Applications, vol. 340, no. 2, pp. 1088-1095, 2008.

[17] B. S. Thakur, D. Thakur, and M. Postolache, "A new iterative scheme for numerical reckoning fixed points of Suzuki's generalized nonexpansive mappings," Applied Mathematics and Computation, vol. 275, pp. 147-155, 2016.

[18] J. Schu, "Weak and strong convergence to fixed points of asymptotically nonexpansive mappings," Bulletin of the Australian Mathematical Society, vol. 43, no. 1, pp. 153-159, 1991.

[19] K. Ullah and M. Arshad, "New three step iteration process and fixed point approximation in Banach space," Journal of Linear and Topological Algebra, vol. 7, no. 2, pp. 87-100, 2018. 
[20] A. M. Harder, Fixed point theory and stability results for fixed point iteration procedures, Ph.D. thesis, University of Missouri, Rolla, MO, USA, 1987.

[21] M. O. Osilike, "Some stability results for fixed point iteration procedures," Journal of the Nigerian Mathematical Society, vol. 14/15, pp. 17-29, 1995.

[22] B. E. Rhoades, "Fixed point theorems and stability results for fixed point iteration procedures II," Indian Journal of Pure and Applied Mathematics, vol. 24, pp. 691-703, 1993.

[23] A. M. Harder and T. L. Hicks, "Stability results for fixed point iteration procedures," Mathematica Japonica, vol. 33, pp. 693-706, 1988.

[24] O. Christopher, Imoru, and O. Olatinwo, "On the stability of Picard and Mann iteration processes," Carpathian Journal of Mathematics, vol. 19, pp. 155-160, 2003.

[25] V. Berinde, "On the stability of some fixed point procedures," Buletinul ştiinţific al Universitatii Baia Mare, Seria B, Fascicola matematică-Informatică, vol. XVIII, no. Nr. 1, pp. 7-14, 2002.

[26] V. Berinde, "Picard iteration converges faster than Mann iteration for a class of quasicontractive operators," Fixed Point Theory and Appl.vol. 2004, p. 1, 2004. 